\title{
Tensile Behavior of Some Mathematical Models of Paper Networks*
}

\author{
Jack C. Smith \\ Center for Materials Science, National Bureau of Standards, Washington, DC 20234 \\ April 20, 1979
}

\begin{abstract}
The tensile behavior of a thin web-like paper network was simulated by two simple mathematical models. The mesh distortion, drop in tensile force and energy loss resulting from breakage of a network junction were calculated. These results were used to formulate two parameters for characterizing interfiber adhesion: a parameter averaging the network energy losses incurred in a series of bond breaks when the network is elongated and a parameter averaging the force drops. The effect of mesh size, local bond adhesive force, and size and shape of the specimen network were calculated. These results based on model studies were used to interpret behavior observed in an actual paper network.
\end{abstract}

Key words: Mathematical modeling, network; network, tensile properties; paper, interfiber bonding; paper, lowdensity handsheets; paper, pulp characterization; paper, tensile testing.

\section{Introduction}

There is a need to develop parameters that can be used to characterize quantitatively the adhesion between fibers in a sheet of paper. Such parameters would be useful in selecting, blending and treating batches of pulp to produce a paper with improved interfiber adhesion. This could facilitate the recycling of paper pulps, leading to important savings in energy and materials.

As a result of some recent research $[1-3]^{1}$ a new technique is evolving for obtaining these parameters. A handsheet in the form of a very low-density web is made from the pulp to be evaluated. If a specimen from this handsheet is elongated to break in a sensitive tensile tester, a force-elongation curve containing numerous jags is obtained. It is assumed that each jag is caused by the breakage of a bond between fibers constituting the handsheet network. Thus if the values of the change in force corresponding to each jag are averaged, a force drop parameter characteristic of interfiber adhesion might be obtained. The energy lost by the network as the result of a bond break can be found by integrating under the force-elongation curve in the vicinity of a jag. An average of these energy losses could also be used as a measure of bond adhesion between two fibers.

The selection of these parameters was an intuitive process. Their applicability must be judged on the basis of experimental evidence. Moreover, what the parameters actually measure is not well understood. Some questions that arise are: How is the strain energy distributed throughout the network after a bond break? How are the energy loss and force drop related to the local force at the bond just before break? What is the effect of mesh size? What is the optimum specimen size and shape for tensile tests to determine the characteristic energy?

In the following, two crude mathematical models of a fibrous network are described, and their behavior under elongation calculated. The concepts developed and the results obtained add to an intuitive understanding of the force drop and energy parameters.

\section{The Parallel-Spring Model}

The parallel-spring model is depicted schematically in figure 1. It consists of a number of springs in a series-parallel arrangement. The dots indicate where the springs are bonded to each other and to a series of

\footnotetext{
* This work was sponsored by the U. S. Department of Energy.

1 Figures in brackets indicate literature references at the end of this paper.
} 


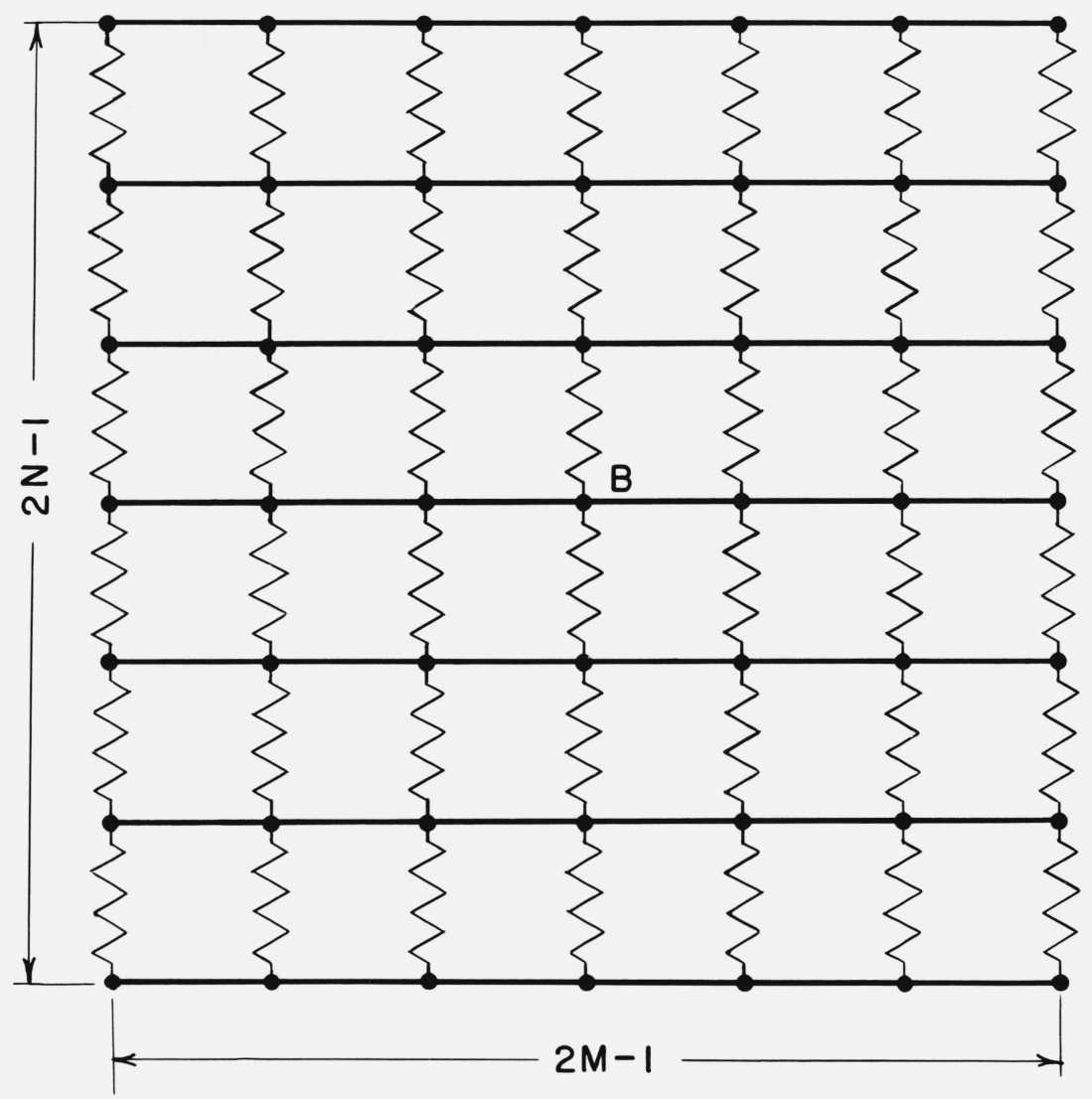

FIGURE 1. Schematic representation of parallel-spring model.

Model has $2 M-1$ parallel columns of springs attached to $2 N-1$ rigid transverse bars.

rigid bars. There are $2 M-1$ junction points on each bar, and there are $2 N-1$ bars. In the unstrained state each spring has a length $l$, called the mesh length. The spring constant is given by $k / l$. The model is elongated so that each spring is extended an amount $l \delta$, where $\delta$ is the strain. The bond $\mathrm{B}$ then breaks, and the two springs previously joined at that point are inactivated.

The initial force sustained by the model before break is

$$
F_{b}=(2 M-1) k \delta
$$

The initial elongation of the model is

$$
\Delta L=2(N-1) l \delta
$$

The initial energy stored in the model is

$$
\begin{aligned}
E_{b} & =1 / 2(2 M-1) k \delta \cdot 2(N-1) l \delta \\
& =2(N-1)(2 M-1) l \cdot 1 / 2 k \delta^{2}
\end{aligned}
$$

After the break the elongation of the model is maintained constant, and the force drops to a value $F_{a}$. The horizontal bars to which the springs are attached remain horizontal. In the two segments of the model on either side of the broken bond the force $F_{a}$ is sustained by $2 M-2$ parallel springs, so that

$$
F_{a}=(2 M-2) k \frac{(\Delta L)_{1}}{l}
$$


or

$$
(\Delta L)_{1}=\frac{F_{a} l}{(2 M-2) k}
$$

where $(\Delta L)_{1}$ is the elongation of the segment.

The elongation in the other $2(N-2)$ segments is given by

$$
(\Delta L)_{2}=\frac{F_{a} l}{(2 M-1) k}
$$

where $(\Delta L)_{2}$ is the elongation of the segment.

The total elongation of all the segments is

$$
2(N-1) l \delta=\frac{2(N-2) F_{a} l}{(2 M-1) k}+\frac{2 F_{a} l}{(2 M-2) k}
$$

Thus

$$
(2 M-1) k \delta=F_{b}=\left\{\frac{N-2}{N-1}+\frac{2 M-1}{2(M-1)(N-1)}\right\} F_{a}
$$

or

$$
\begin{aligned}
F_{b} & =\left\{1+\frac{2 M-1}{2(M-1)(N-1)}-\frac{1}{N-1}\right\} F_{a} \\
& =\left\{1+\frac{1}{2(M-1)(N-1)}\right\} F_{a}
\end{aligned}
$$

Hence

$$
F_{a}=\bar{T} F_{b}
$$

where

$$
\bar{T}=\frac{2(M-1)(N-1)}{2(M-1)(N-1)+1}
$$

$\bar{T}$ is the fraction of the force remaining after a bond break. The force drop resulting from the break is given by

$$
\Delta F=F_{b}(1-\bar{T})
$$

The energy in the two segments of the model adjoining the broken bond is

$$
1 / 2(\Delta L)_{1} \cdot F_{a}=1 / 2 \frac{F_{a}{ }^{2} l}{(2 M-2) k}
$$

and the energy in the other $2(N-2)$ segments is

$$
1 / 2(\Delta L)_{2} \cdot F_{a}=1 / 2 \frac{F_{a}{ }^{2} l}{(2 M-1) k}
$$


Thus the total energy is

$$
\begin{aligned}
E_{a} & =2(N-2) \cdot 1 / 2 \frac{F_{a}{ }^{2} l}{(2 M-1) k}+2 \cdot 1 / 2 \frac{F_{a}{ }^{2} l}{(2 M-2) k} \\
& =2\left\{\frac{N-2}{2 M-1}+\frac{1}{2 M-2}\right\} \cdot 1 / 2 \frac{F_{a}{ }^{2} l}{k} \\
& =\frac{2(N-1)}{2 M-1}\left\{\frac{N-2}{N-1}+\frac{2 M-1}{2(M-1)(N-1)}\right\} \frac{l}{2 k} \cdot \bar{T}^{2} \cdot(2 M-1)^{2} k^{2} \delta^{2} \\
& =2(N-1)(2 M-1) l \cdot \frac{1}{\bar{T}} \cdot \bar{T}^{2 \cdot 1 / 2} k \delta^{2}
\end{aligned}
$$

or

$$
\begin{aligned}
E_{a} & =2(N-1)(2 M-1) l \bar{T} \cdot 1 / 2 k \delta^{2} \\
& =\bar{T} E_{b}
\end{aligned}
$$

In order to describe the way strain energy is distributed in a model it is useful to associate energy with each of the junction points in such a way that the sum of the associated energies is equal to the total energy stored. At any given junction point the associated energy is equal to one-half the sum of the energies in the springs joined at that point. The factor of one-half is introduced because when the associated energies are summed over all the junction points, the energy of each spring is counted twice. At junction points on the top and bottom boundaries there is only one spring, but the factor of one-half is still necessary.

A dimensionless average associated energy $\bar{E}$ can be defined by means of the relation,

$$
E_{a}=(2 M-1)(2 N-1) \bar{E} \cdot 1 / 2 k \delta^{2} l
$$

The factor $(2 M-1)(2 N-1)$ gives the total number of points in the network. The factor $1 / 2 k \delta^{2} l$ is the energy stored in one spring before a bond break, and is introduced into the expression to make $\bar{E}$ dimensionless. By equating relations (1.9) and (1.10) an alternative relation,

$$
\bar{E}=\frac{2 N-2}{2 N-1} \bar{T}
$$

is obtained.

The energy loss resulting from a bond break is, from eq (1.9),

$$
\Delta E=E_{b}-E_{a}=(1-\bar{T}) E_{b}
$$

An alternative form is obtained from eqs (1.3) and (1.10).

$$
\Delta E=2(N-1)(2 M-1) l\left(1-\frac{2 N-1}{2 N-2} \bar{E}\right) \cdot 1 / 2 k \delta^{2}
$$

\section{The Square-Network Model}

The square-network model is depicted schematically in figure 2. It consists of springs forming a square network. There are $2 M-1$ columns of junction points and $2 N-1$ rows of junction points. The depicted network has 7 columns $(M=4)$ and 7 rows $(N=4)$. The unstrained length of each spring is $l$, and the spring constant is given by $k / l$. The model is elongated by an amount $2(N-1) l \delta$. The bond at point $\mathrm{B}$ then breaks, and the four springs previously joined at that point are inactivated. The resulting configuration is calculated by a computer program described below. 


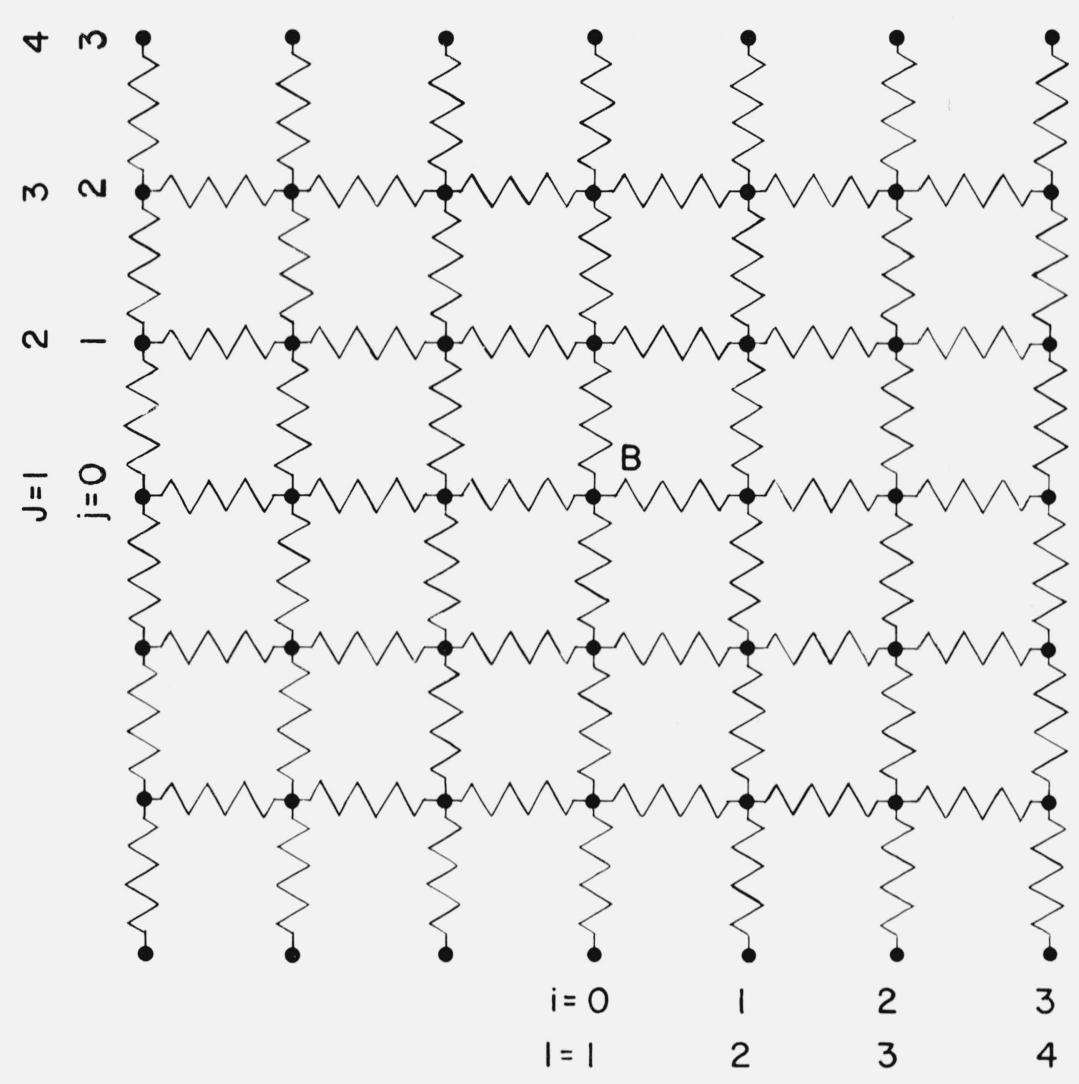

FIGURE 2. Schematic representation of square-network model.

Model has $2 M-1$ parallel columns of springs. Each column has $2 N-1$ junction points which, excepting the end points, are attached to transverse springs.

In order to discuss the model it is convenient to identify junction points by the coordinates $i=0,1, \cdots m$; $j=0,1, \cdots n$. The corresponding numbers $I=1,2, \cdots M ; J=1,2, \cdots N$, however, are retained for use in the formulas for force and energy, as they are more convenient for counting purposes. Because of symmetry it is only necessary to calculate the configuration of the upper right-hand quadrant; i.e., the junction points corresponding to positive values of $i$ and $j$.

When the model is strained each of the junction points moves from its original position by an amount $\xi_{i j} l$ in the horizontal direction and $\eta_{i j} l$ in the vertical direction. Thus when the model is first extended and no bond break has occurred,

$$
\left.\begin{array}{rl}
\xi_{i j} & =0 . \\
\eta_{i j} & =j \delta
\end{array}\right\}
$$

After the bond break the values of $\xi_{i j}, \eta_{i j}$ readjust except for certain constraints at the boundary and the $i=0, j=0$ axes of symmetry. These are

$$
\left.\begin{array}{c}
\xi_{i n}=0 . \\
\eta_{i n}=n \delta
\end{array}\right\}
$$




$$
\left.\begin{array}{rl}
\xi_{-1 j} & =-\xi_{1 j} \\
\eta_{-1 j} & =\eta_{1 j}
\end{array}\right\}
$$

The values of $\xi, \eta$ attained after bond break are found by solving a system of equations in which the horizontal and vertical forces at each junction point are balanced out, plus the boundary conditions (2.2-2.6). To formulate the equations applicable at the point $i, j$, let

$$
\left.\begin{array}{l}
R_{A}=\sqrt{\left(1+\Delta_{A} \xi\right)^{2}+\Delta_{A} \eta^{2}} \\
R_{B}=\sqrt{\Delta_{B} \xi^{2}+\left(1+\Delta_{B} \eta\right)^{2}} \\
R_{C}=\sqrt{\Delta_{C} \xi^{2}+\left(1+\Delta_{C} \eta\right)^{2}} \\
R_{D}=\sqrt{\left(1+\Delta_{D} \xi\right)^{2}+\Delta_{D} \eta^{2}}
\end{array}\right\}
$$

where $\Delta_{A}$ is a difference operator involving the indices $(i, i-1), \Delta_{B}$ the indices $(j, j-1), \Delta_{C}$ the indices $(j+1, j)$ and $\Delta_{D}$ the indices $(i+1, i)$. Thus, for instance, $\Delta_{A} \xi$ means $\xi_{i j}-\xi_{i-1, j}$. The quantities $R l$ are the distances from a point $i j$ to an adjacent point. The horizontal force balance equation is then

$$
\frac{k\left(R_{D}-1\right)\left(1+\Delta_{D} \xi\right)}{R_{D}}+\frac{k\left(R_{C}-1\right) \Delta_{C} \xi}{R_{C}}=\frac{k\left(R_{B}-1\right) \Delta_{B} \xi}{R_{B}}+\frac{k\left(R_{A}-1\right)\left(1+\Delta_{A} \xi\right)}{R_{A}}
$$

or

$$
\left(1+\Delta_{D} \xi\right)\left(1-1 / R_{D}\right)+\Delta_{C} \xi\left(1-1 / R_{C}\right)-\Delta_{B} \xi\left(1-1 / R_{B}\right)-\left(1+\Delta_{D} \xi\right)\left(1-1 / R_{D}\right)=0 .
$$

Similarly for the vertical components

$$
\Delta_{D} \eta\left(1-1 / R_{D}\right)+\left(1+\Delta_{C} \eta\right)\left(1-1 / R_{C}\right)-\left(1+\Delta_{B} \eta\right)\left(1-1 / R_{B}\right)-\Delta_{A} \eta\left(1-1 / R_{A}\right)=0 .
$$

Equations (2.8) and (2.9) apply at the general junction point $i j$, but when $i=0, m$ or $j=0, n$ they must be modified to conform with boundary or symmetry conditions.

It should be noted that the horizontal forces acting at a point $i, j$ are dependent mostly upon the values of $\xi_{i-1, j}, \xi_{i j}$ and $\xi_{i+1, j}$. Therefore eq (2.8) which has the form

$$
f\left(\xi_{i-1, j}, \eta_{i-1, j}, \xi_{i j}, \eta_{i j}, \xi_{i+1, j}, \eta_{i+1, j}, \xi_{i, j-1}, \eta_{i, j-1}, \xi_{i, j+1}, \eta_{i, j+1}\right)=0 .
$$

was expressed as a Taylor's series expansion in terms of only these three most important variables to become

$$
f^{(k)}+\left(\frac{\partial f}{\partial \xi_{i-1, j}}\right)^{(k)} \Delta \xi_{i-1, j}+\left(\frac{\partial f}{\partial \xi_{i j}}\right)^{(k)} \Delta \xi_{i j}+\left(\frac{\partial f}{\partial \xi_{i+1, j}}\right)^{(k)} \Delta \xi_{i+1, j}=0
$$

where the $\Delta \xi_{i j}$ means $\xi_{i j}{ }^{(k+1)}-\xi_{i j}{ }^{(k)}$ and $\Delta \xi_{i-1, j}$ and $\Delta \xi_{i+1, j}$ have similar meanings. $f^{(k)},\left(\frac{\partial f}{\partial \xi_{i j}}\right)^{(k)}$ etc. are evaluated as functions of $\xi^{(k)}, \eta^{(k)}$. The values of $\xi^{(k+1)}$ are obtained by solving the system of eqs (2.11) corresponding to different values of $j$.

A set of five of these equations, for instance, would look like

$$
\begin{aligned}
B_{1} x_{1}+C_{1} x_{2} & =K_{1} \\
A_{2} x_{1}+B_{2} x_{2}+C_{2} x_{3} & =K_{2} \\
A_{3} x_{2}+B_{3} x_{3}+C_{3} x_{4} & =K_{3} \\
A_{4} x_{3}+B_{4} x_{4}+C_{4} x_{5} & =K_{4} \\
A_{5} x_{5}+B_{5} x_{5} & =K_{5}
\end{aligned}
$$


This set of equations is easily solved by a Gauss-Jordan reduction.

Equations (2.11) are solved to obtain values of $\xi^{(k+1)}$. Similarly eq (2.9) can be expanded in terms of $\eta_{i, j-1}, \eta_{i j}$ and $\eta_{i, j+1}$. The set of equations obtained for different values of $i$ can be solved to obtain values of $\eta^{(k+1)}$. The values of $f,\left(\frac{\partial f}{\partial \xi_{i j}}\right),\left(\frac{\partial f}{\partial \eta_{i j}}\right)$ in eq (2.11) for $\xi$ and the corresponding equation for $\eta$ are then evaluated using $\xi^{(k+1)}, \eta^{(k+1)}$ and the procedure iterated to obtain $\xi^{(k+2)}, \eta^{(k+2)}$.

During the calculation process solutions for $\xi$ and $\eta$ may be obtained such that $R_{A}, R_{B}, R_{C}$, or $R_{D}$ becomes less than 1 in value. This means that there is a spring in compression. This situation is not permitted as it is more realistic to assume that the spring would buckle rather than resist a compressive force. Therefore if there is an $R$ less than 1, modified equations in which the influence of the spring is removed are used in an alternate calculation.

The configuration of the network can be calculated in other ways. For instance eqs (2.8) and (2.9) could be expanded in the variables $\xi_{i j}, \eta_{i j}$. Improved approximations for $\xi, \eta$ could be obtained by solving only two simultaneous equations at each point, but a very large number of iterations would be required to obtain very precise values of $\xi, \eta$. On the other hand the equations could be expanded in terms of all 10 variables. In this way a system of approximately $M N$ equations in $M N$ unknowns would be obtained. If these equations had a numerically stable solution and round-off errors were not too severe, only a few iterations might be required.

The method of solution that was used was a compromise between these two extremes. It appears to be stable but in most cases requires 100 or 200 iterations to obtain satisfactorily precise values. When situations are calculated in which the initial strain $\delta$ is of the order of one percent, more than 200 iterations may be required. In this situation the values of some $R \mathrm{~s}$ are very close to unity and very small values of $R-1$ are obtained. Thus it might be better in this case to rewrite eqs (2.8) and (2.9), expressing terms of the form $(1-1 / R)$ as a truncated series, although this was not done for the calculations reported here.

After sufficiently accurate values of $\xi$ and $\eta$ were obtained, the tensile force $T_{i n}$ at the top of each column of springs was calculated using the formula

$$
\left.\begin{array}{l}
T_{i n}=k\left(1+n \delta-\eta_{i, n-1}\right)\left(1-1 / R_{B}\right) \\
R_{B}=\sqrt{\xi_{i, n-1}^{2}+\left(1+n \delta-\eta_{i, n-1}\right)^{2}}
\end{array}\right\}
$$

When $i=0$, the value of $T_{0 n}$ simplifies to

$$
T_{0 n}=k\left(n \delta-\eta_{0, n-1}\right)
$$

The force after break $F_{a}$ then becomes

$$
F_{a}=T_{0 n}+2 \sum_{i=1}^{n} T_{i n}
$$

The fraction of the force remaining after a bond break $\bar{T}$ is given by the ratio $F_{a} / F_{b}$.

The average associated energy $\bar{E}$ is found from its definition, eq (1.10), after calculating the associated energy for each junction point and summing over all the junction points to obtain the stored energy after break $E_{a}$.

The value of $\bar{E}$ can be obtained by a different calculation, if $F_{a}$ is known as a function of $\delta$ from a series of calculations on a given model. The energy after break $E_{a}$ is found by integrating under the curve of $F_{a}$ versus initial elongation $\Delta L=2(N-1) l \delta$. Thus,

$$
\begin{aligned}
E_{a} & =\int_{0}^{2(N-1) l \delta} F_{a} \cdot d[2(N-1) l \delta] \\
& =2(N-1)(2 M-1) k l \int_{0}^{\delta} \bar{T} \delta d \delta
\end{aligned}
$$


From the definition eq (1.10) $\bar{E}$ is then given by

$$
\bar{E}=\frac{2 N-2}{2 N-1} \cdot \frac{2}{\delta^{2}} \int_{0}^{\delta} \bar{T} \delta d \delta
$$

In the square-network model $\bar{T}$ is not a constant independent of $\delta$ as it is in the parallel-spring model. If $\bar{T}$ were constant, eq (2.16) would reduce to the relation eq (1.11) given for the parallel-spring model.

It should be noted that the behavior of the square-network model is the same as that of the parallel-spring model before the central bond breaks. Therefore eqs (1.1), (1.2) and (1.3) are the same for both models. The quantity $\bar{T}$ is defined by eq (1.6) and the quantity $\bar{E}$ by eq (1.10) in both models. Therefore eqs (1.8) and (1.13) are valid for both models. Relation (2.16) is a general definition valid for both models, but relations (1.7) and (1.11) apply to the parallel-spring model only. Therefore eqs (1.9) and (1.12) also are valid only for the parallel-spring model.

\section{Square-Network Model Calculations}

A number of calculations were carried out for different square-network models to determine the number of iterations required for accurate values of $\bar{T}$ and $\bar{E}$. The model for which $M=11, N=21, l=1$ was studied the most extensively. Results of calculations for this model are given in table 1 . These results indicate that for

TABLE 1. Values of $\bar{T}$ and $\bar{E}$ as Functions of Number of Iterations for a Square-Network Model $M=11, N=21, l=1$.

\begin{tabular}{c|c|c|c}
\hline \hline$\delta$ & $\begin{array}{c}\text { No. of } \\
\text { Iterations }\end{array}$ & $\bar{T}$ & $\bar{E}$ \\
\hline \multirow{2}{*}{0.01} & 100 & 0.95227 & 0.92923 \\
& 200 & .95254 & .92923 \\
& & & .93112 \\
& 100 & .95779 & .93100 \\
& 200 & .95714 & .93606 \\
& & & .93583 \\
& 20 & .96738 & .93567 \\
& 50 & .96679 & .93561 \\
& 100 & .96614 & .94643 \\
& 200 & .96564 & .94638 \\
& & & .94637 \\
& 20 & .97806 & .95270 \\
& 50 & .97829 & .95263 \\
& 100 & .97827 & .95262 \\
& 20 & .98316 & .97979 \\
& 50 & .98330 & .95679 \\
& 100 & .98332 & .95644 \\
& 10 & .95641 & .95640 \\
\hline
\end{tabular}

TABLE 2. Values of $\bar{T}$ and $\bar{E}$ for Some Square-Network Models $\delta=0.20, l=1$, 200 Iterations.

\begin{tabular}{c|c|c|c}
\hline \hline$M$ & $N$ & $\bar{T}$ & $\bar{E}$ \\
\hline 11 & 11 & 0.97554 & 0.92306 \\
11 & 21 & .98597 & .95640 \\
21 & 21 & .99311 & .96639 \\
11 & 41 & .99238 & .97634 \\
21 & 41 & .99626 & .98221 \\
\hline
\end{tabular}


values of $\delta$ greater than $0.05,100$ or 200 iterations are required to obtain values of $\bar{T}$ and $\bar{E}$ accurate to more than four significant digits. When $\delta$ is 0.05 or less, more than 200 iterations are desirable. Check calculations were also carried out on the other models studied, and 200 iterations were determined as sufficient.

Calculations were carried out on five different models. The initial strain $\delta$ for these models was 0.20 . A mesh length of $l=1$ was assumed for the calculations. Values of $M$ and $N$ were varied in order to study how $\bar{T}$ and $\bar{E}$ depended on the shape and on the number of junctions in the model. Results of the calculations for these models are given in table 2.

The configuration of the model for which $M=11, N=21$ is plotted in figure 3. Only the upper right-hand quadrant of the model is shown, as the complete model is symmetrical with respect to this quadrant. The distortion due to the bond break is seen to be concentrated along the central column of springs $(I=1)$. Buckling occurs $(R<1)$ in the regions where the springs are indicated by dotted lines. Although not easily discernable in the figure, the central width $(J=1)$ has been reduced to 0.956 times the original width, as a result of bond break.

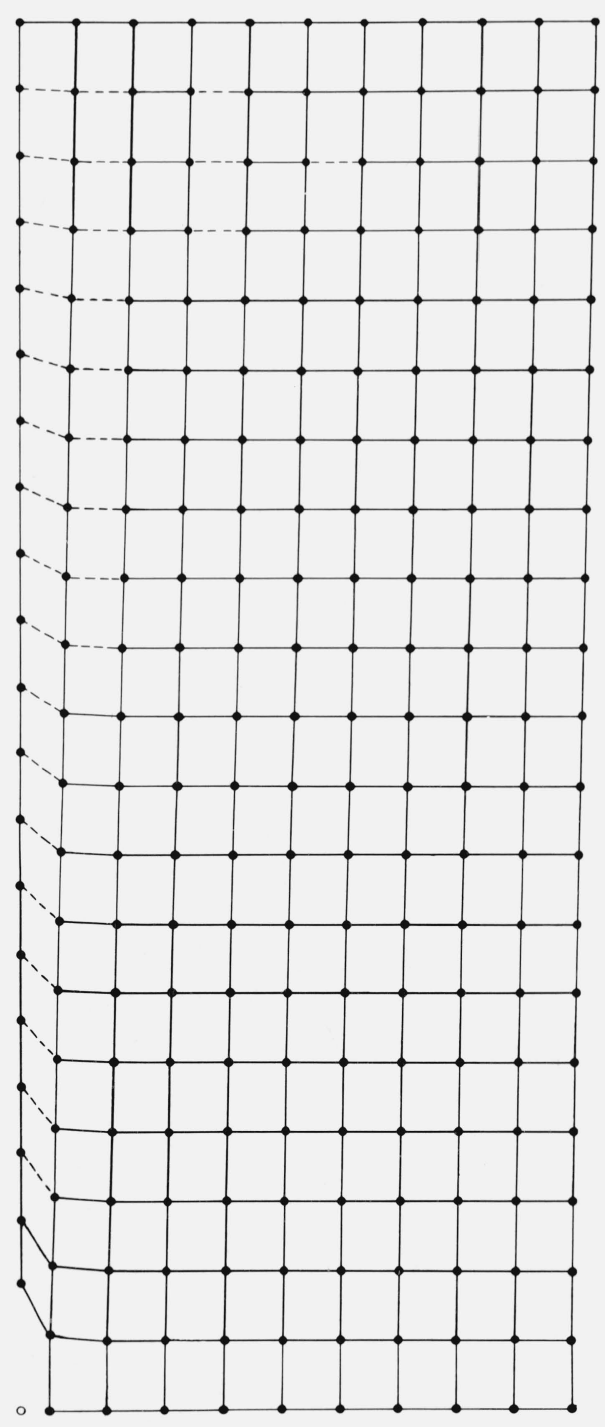

Figure 3. Configuration of the positive quadrant of a square-network model for which $M=11$, $N=21$, after a central bond break.

The broken bond is designated by the open circle in the lower left corner. Springs in which buckling occurs are designated by dashed lines. 


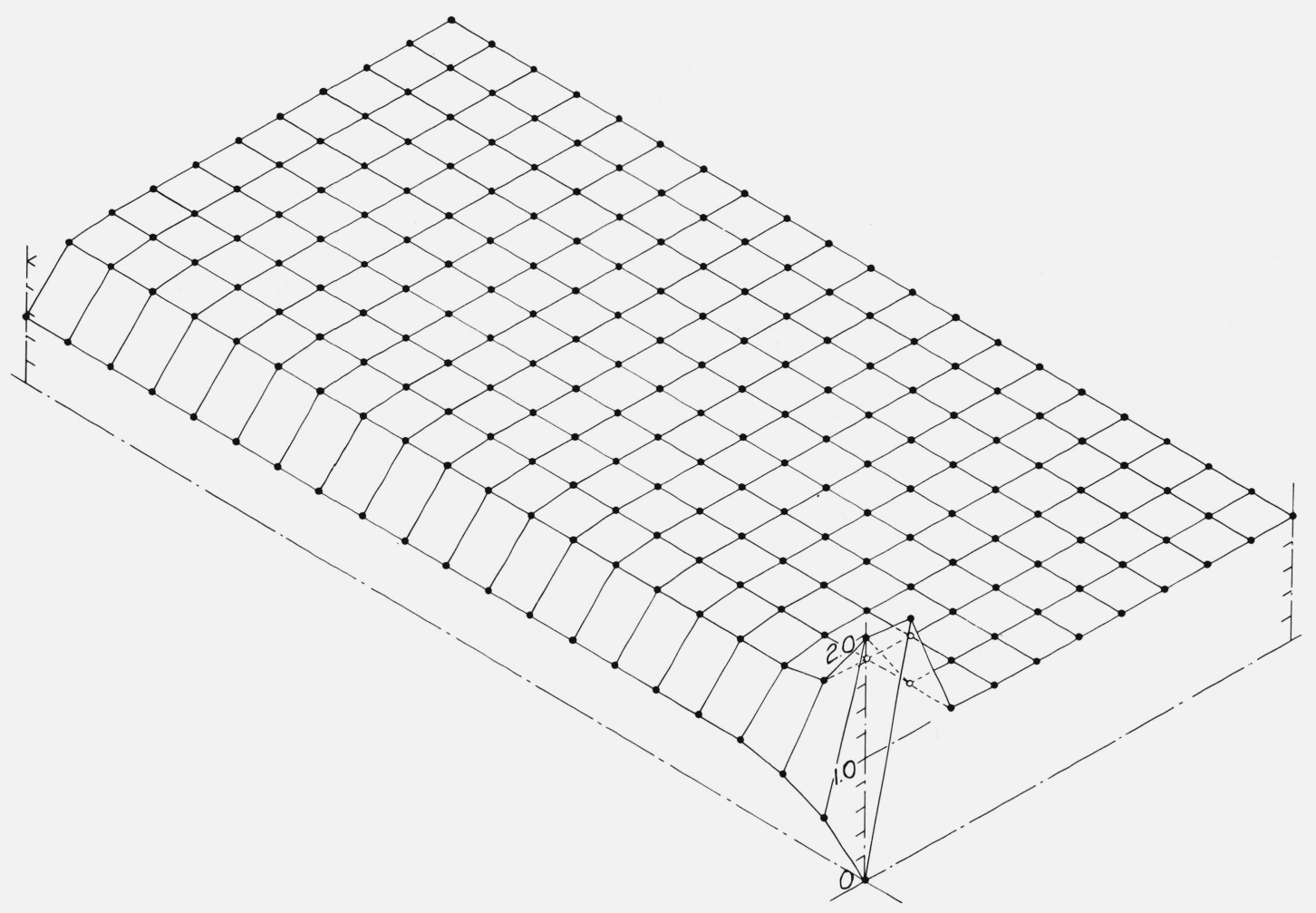

Figure 4. Energy map of positive quadrant of a square-network model for which $M=11, N=21$, after a central bond break. Vertical-axis scale gives ratio of associated energy at a junction point after bond break, divided by the associated energy before break.

TABLE 3. Values of $\bar{T}$ and $\bar{E}$ as Functions of Initial Strain for a Square-Network Model

\begin{tabular}{c|c|c|c}
\multicolumn{4}{c}{$M=11, N=21, l=1}$. \\
\hline \hline$\delta$ & $\bar{T}$ & $\bar{E}$ & $\begin{array}{c}\bar{E} \\
\text { eq }(2.16)\end{array}$ \\
\hline 0.01 & 0.95254 & 0.92923 & 0.92931 \\
.03 & .95714 & .93100 & .93230 \\
.05 & .96564 & .93561 & .93658 \\
.08 & .97477 & .94270 & .94328 \\
.10 & .97827 & .94637 & .94683 \\
.13 & .98173 & .95052 & .95078 \\
.15 & .98332 & & .95273 \\
.18 & .98507 & .95262 & .95503 \\
.20 & .98597 & .95510 & .95626 \\
\hline
\end{tabular}

Note: $\bar{T}$ for the parallel-spring model is 0.99751

$\bar{E}$ for the parallel-spring model is 0.97318

Note: Values for ${ }^{\circ}=0.01,0.03,0.05$ and 0.20 were obtained after 200 iterations. All other values were obtained after 100 iterations.

An energy map of the model for which $M=11, N=21$ is shown as an isometric plot in figure 4 . As before only the upper right-hand quadrant of the model is shown. The dimensionless value plotted at each junction point is the ratio of the associated energy after bond break to the associated energy before bond break. According to this map the associated energy remains constant at most of the junction points, it increases at points immediately to the right and left of the broken bond and decreases to a lower level at junction points above and below the broken bond. 


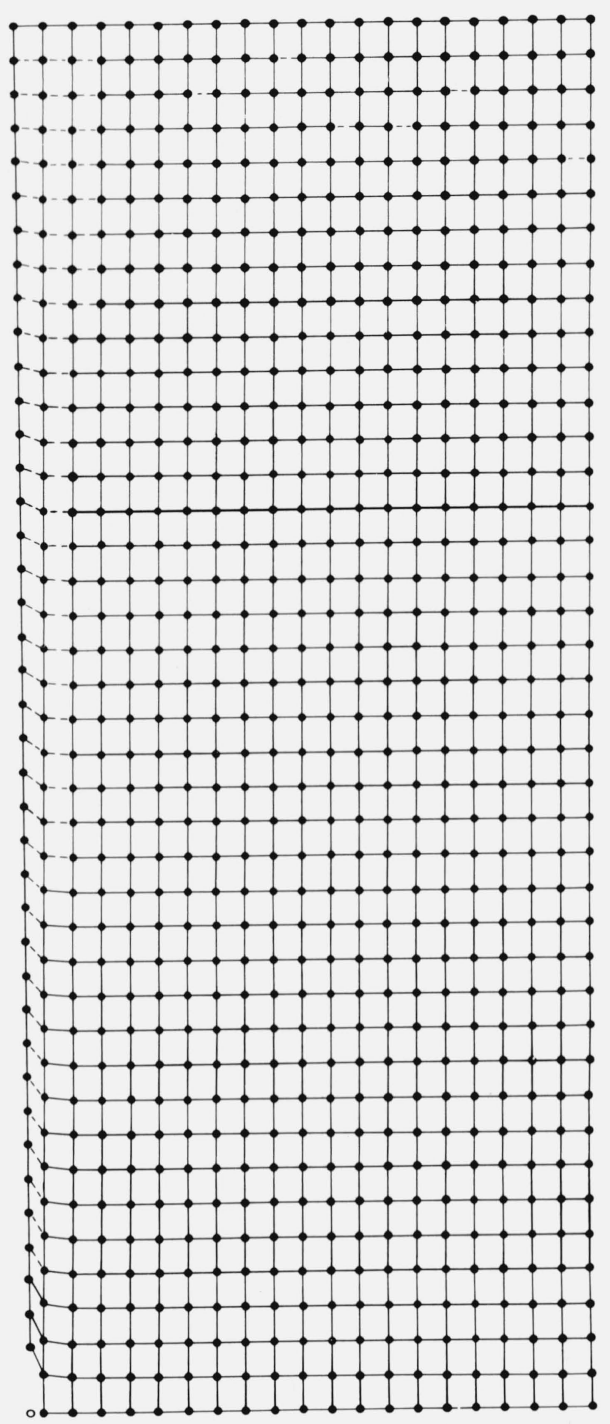

Figure 5. Configuration of the positive quadrant of a square-network model for which $M=21$, $N=41$, after a central bond break.

The broken bond is designated by the open circle in the lower left corner. Springs in which buckling occurs are designated by dashed lines.

Figure 5 shows the configuration after bond break, and figure 6 shows the energy map of the model for which $M=21, N=41$. This model, of the same shape as the previous model, has approximately four times as many junction points. The corresponding figures for the two models are similar.

Table 3 presents values of $\bar{T}$ and $\bar{E}$ as functions of initial strain $\delta$ in the case of the model for which $\boldsymbol{M}=11, N=21$. A force-elongation curve for this model after one central bond has been broken can be plotted using data from table 3 . The force has the value $(2 M-1) \bar{T} k \delta$, and elongation the value $2(N-1) l \delta$. The essential characteristics of the curve are shown by figure 7 , which is a plot of $\bar{T} \delta$ vs $\delta$ for this model. Unfortunately the values of $\bar{T}$ are so close to unity that this curve lies very close to the force-elongation curve, shown as a dashed line, of the model before the central bond was broken, and the nonlinear character of the curve is not readily apparent.

The non-linear character of the force-elongation curve can be demonstrated by plotting the difference in force before and after break as a function of $\delta$. This is shown in figure 8 , in which $(1-\bar{T}) \delta$ is plotted vs $\delta$. As 


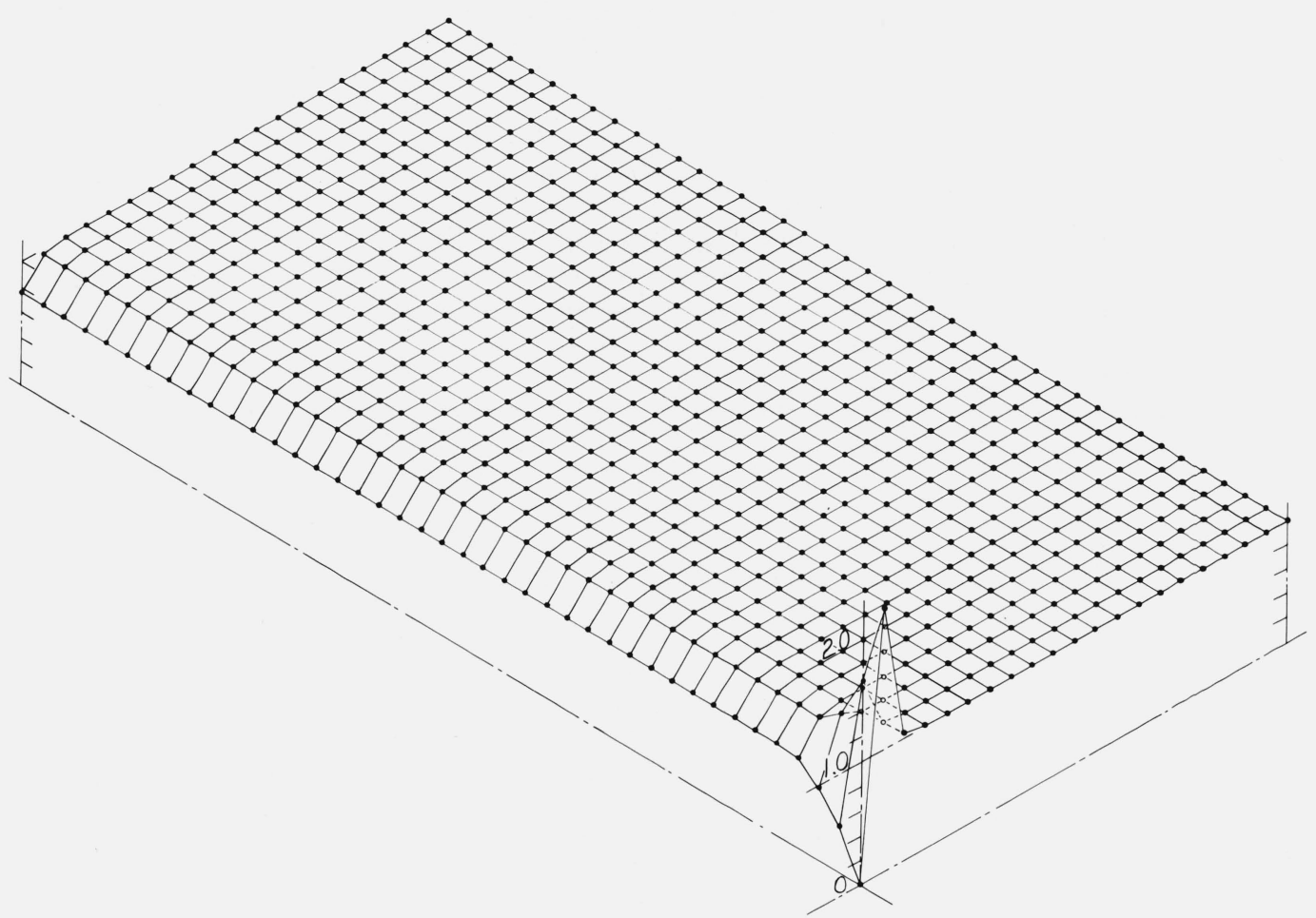

FigURE 6. Energy map of positive quadrant of a square-network model for which $M=21, N=41$, after a central bond break. Vertical-axis scale gives ratio of associated energy at a junction point after bond break, divided by the associated energy before break.

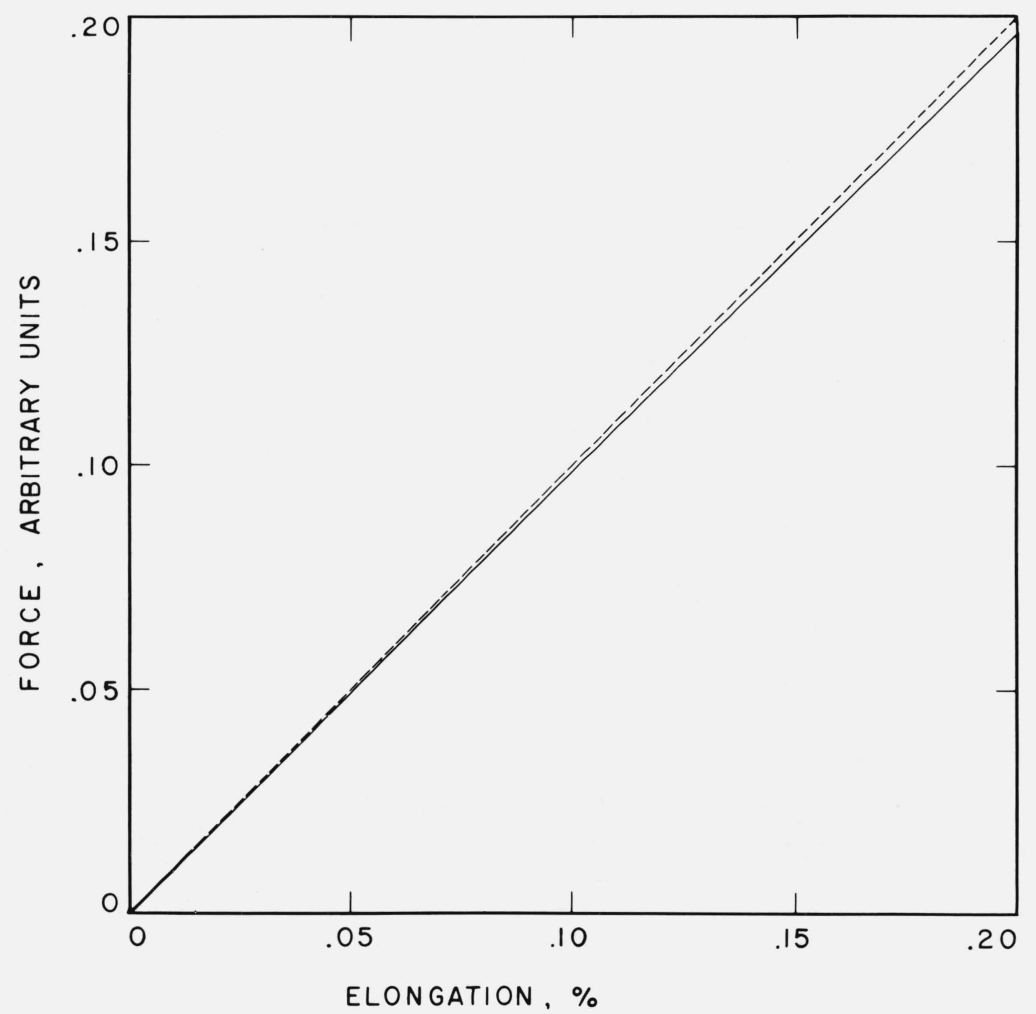

FIGURE 7. Force-elongation curve after central bond break in a square-network model for which $M=11, N=21$. $\bar{T} \delta$ is plotted vs $\delta$, where $\delta$ is the elongation per unit length. Dashed line shows force-elongation curve before bond break. 


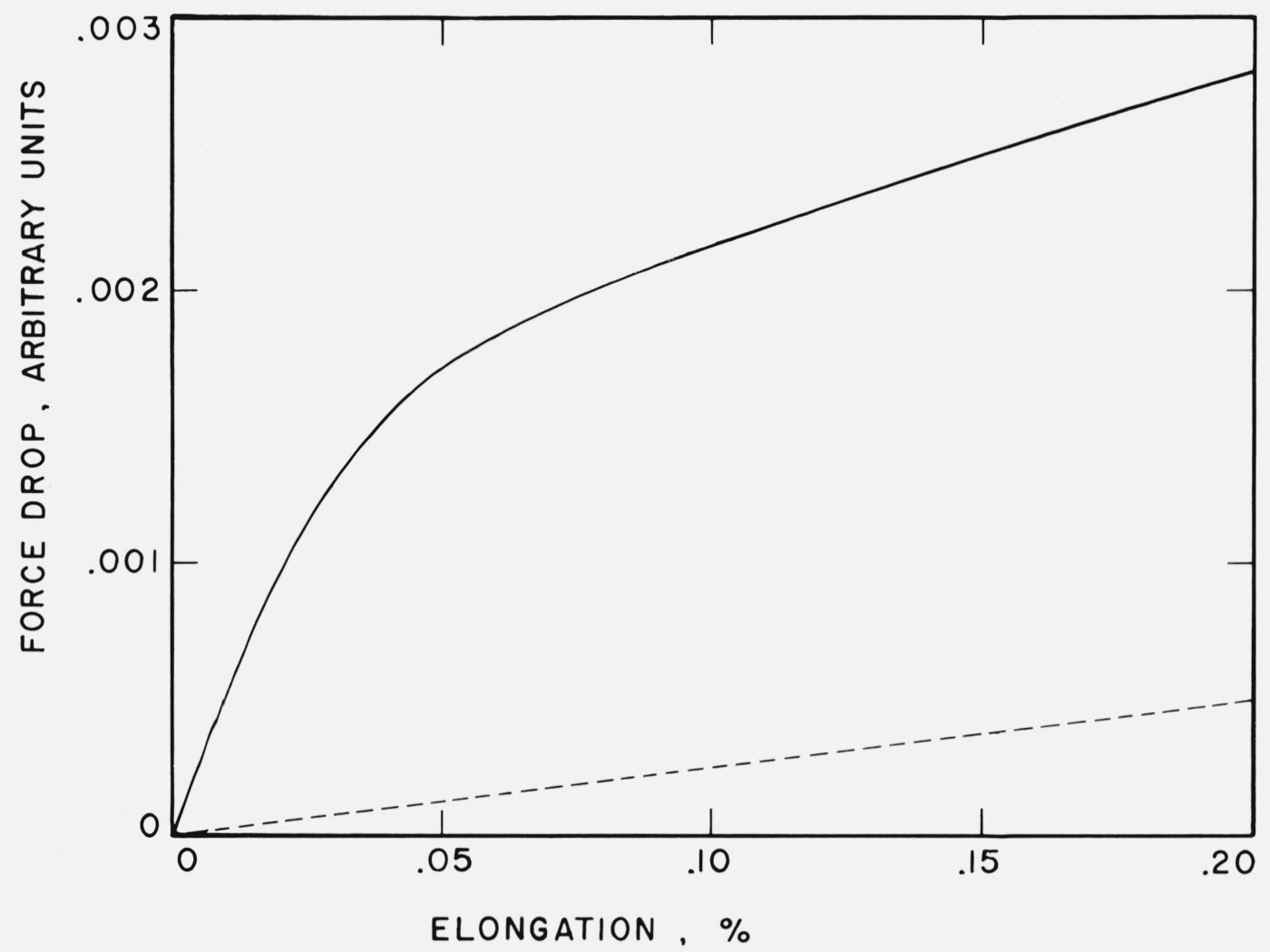

Figure 8. Curve of force drop due to bond break vs elongation of a square-network model for which $M=11, N=21$. $(1-\bar{T}) \delta$ is plotted vs $\delta$, where $\delta$ is the elongation per unit length. Dashed line shows force drop for equivalent parallel-spring model.

a matter of interest $(1-\bar{T})$ for the parallel-spring model $(M=11, N=21)$ was calculated. Its value was 0.0024938 . The linear function $0.0024938 \delta$ is shown as a dashed line on the plot. The value of $\bar{T}$ for the parallel-spring model exceeds the values of $\bar{T}$ calculated for the square-network model. Evidently the force drop incurred by a bond break in the square-network model is greater than the force drop incurred in the parallel-spring model.

The area under the $\bar{T} \delta$ curve in figure 7 is equal to $\int_{0}^{\delta} \bar{T} \delta d \delta$, and according to eq (2.16) the average associated $\bar{E}$ is equal to $\frac{2 N-2}{2 N-1} \cdot \frac{2}{\delta^{2}}$ times this quantity. Thus values of $\bar{E}$ can be calculated from $\bar{T}$ and $\delta$ according to eq (2.16) and the result compared with the values of $\bar{E}$ in table 3 , which were calculated from the associated energies present after break. This should provide a check on the accuracy of the computer calculations. The results of this calculation are given in the last column of table 3 . The values of $\bar{E}$ obtained by the two methods are seen to be in fair agreement.

\section{Discussion of Results}

\section{Configurational Distortions and the Redistribution of Associated Energies as the Result of a Bond Break.}

It has been assumed that in a low-density paper network of the type tested experimentally [1-3], in most cases a bond break results only in local distortions, and most of the energy loss is also concentrated locally. It would be desirable to demonstrate this with a mathematical model, but the square-network model is not suitable for this purpose. The distortions and energy losses are concentrated along the column of springs directly in line with the broken bond. Possibly a better model would be one in which the meshes were of a different shape, such as a hexagon, and so arranged that load bearing fibers could not be easily aligned in the direction of stretch. Such a model would require less stretching force, have a nonlinear force-elongation 
curve, and probably have a greater tendency to buckle laterally. It would better simulate the behavior of a thin paper network, but at the price of greater mathematical complexity.

In the energy maps, figures 4 and 6 , there is an increase in associated energy at the junctions to either side of the broken bond. These bonds therefore should break immediately after breakage of the central bond, and bonds farther out should break successively in a tearing action that proceeds across the model network. This tearing action is probably common to many models, and actually occurs in real paper networks. In the paper networks however it seldom goes to completion, because the density of fibers is not sufficiently uniform.

\section{The Effect of Mesh Length.}

In experimental work specimens of standard dimensions (usually $2 \times 1 \mathrm{~cm}$ ) are elongated, and the applied force recorded as a function of the elongation. Whenever a bond breaks the force decreases sharply, and the energy loss resulting from the bond break can be determined by integrating under the force-elongation curve in the vicinity of the force drop. Certain of these energy losses are averaged to obtain a parameter characterizing bond strength.

If it is assumed that all of the breaks occur at approximately the same local force level, and the specimens have uniform mesh size, this energy parameter might provide a reliable indication of the relative bond strength. For instance bonding could be studied in thin handsheets of the same mass per unit area made from a pulp that had been subjected to various beating treatments. However if one attempts to compare bonding in standard handsheets made from different pulps, a difficulty arises. One pulp may be coarser than the other. Thus a handsheet of a standard mass per unit area made from a coarse pulp would have fewer bonded junctions and larger meshes on the average than would a handsheet made from a finer pulp. In order to compare energy parameters for these different pulps it is necessary to know what is the effect of mesh size.

To study this effect in models, consider two parallel-spring models: model A; $M=11, N=21, l=1$ and model $\mathrm{B} ; M=21, N=41, l=0.5$. These models have the same external dimensions, but the mesh length of model B is half that of model A. Let the two models be extended the same amount, so that $\delta$ and therefore the local force $k \delta$ is the same in each. From eqs (1.1), (1.7) and (1.8) the force drop in model A is $21 k \delta / 401$ $\sim 0.05 k \delta$, and in model B is $41 k \delta / 1601 \sim 0.026 k \delta$. From eqs (1.3), (1.7) and (1.12) the energy loss in model A is $\frac{840}{401} \cdot 1 / 2 k \delta^{2} \sim 2.1 \cdot 1 / 2 k \delta^{2}$, and in model B is $\frac{1640}{1601} \cdot 1 / 2 k \delta^{2} \sim 1.02 \cdot 1 / 2 k \delta^{2}$. Evidently model B with half the mesh size of model A also has approximately half the force drop and half the energy loss.

In any parallel-spring model, from eqs (1.1), (1.7) and (1.8)

$$
\Delta F=\frac{(2 M-1) k \delta}{2(M-1)(N-1)+1}=\frac{2(M-1)+1}{2(M-1)(N-1)+1} k \delta
$$

which for large $M$ becomes

$$
\Delta F \sim \frac{1}{N-1} k \delta
$$

Substituting the initial length $\mathrm{L}=2(N-1) l$ into this expression gives

$$
\Delta F \sim \frac{2 l}{L} k \delta
$$

From eqs (1.3), (1.7) and (1.12)

$$
\begin{aligned}
\Delta E & =\frac{2(N-1)(2 M-1) l}{2(M-1)(N-1)+1} \cdot \frac{1}{2} k \delta^{2} \\
& =\frac{2(N-1)(2 M-2)+2(N-1)}{2(M-1)(N-1)+1} l \cdot \frac{1}{2} k \delta^{2}
\end{aligned}
$$


which for large $M$ becomes

$$
\Delta E \sim 2 l \cdot 1 / 2 k \delta^{2}
$$

According to eqs (4.1) and (4.2), if any two parallel-spring models for which $M$ is large are extended so that the local force $k \delta$ is the same in each model, the ratio of the energy losses $\Delta E_{1} / \Delta E_{2}$ in the two models will be the same as the ratios of the respective mesh lengths $l_{1} / l_{2}$. If in addition the initial lengths of the two models are the same, i.e., $2\left(N_{1}-1\right) l_{1}=2\left(N_{2}-1\right) l_{2}$, the ratios of the force drops will be equal to $l_{1} / l_{2}$.

Table 4 gives the results of calculations for a number of parallel-spring models, all of which have the same initial length. According to these results the force drops and energy losses are almost linearly proportional to the mesh length $l$, despite the relatively low value of $M$ in some of these models. It is interesting to observe that values of total force before break $F_{b}$ and the total energy before break $E_{b}$ do not influence these results significantly. The values of $F_{b}$ and $E_{b}$ are proportional to $2 M-1$ however, so this is just a manifestation of the near independence of the values of $\Delta F$ and $\Delta E$ on the values of $M$, for $M$ sufficiently large (e.g., $M=11$ ).

Table 5 gives results of calculations for some square-network models having the same initial length. The $M, N$ and $l$ parameters for these models are the same as those in table 4 . The force drops and energy losses for these models decrease as the mesh length $l$ decreases, but are not linearly proportional to the mesh length. For instance the three models for which $M=11$ have $l$ ratios of 1.0:0.50:0.25, $\Delta F$ ratios of 1.0:0.57:0.31 and $\Delta E$ ratios of 1.0:0.64:0.37. The two models for which $M=21$ have $l$ ratios of 1.0:0.50, $\Delta F$ ratios of 1.0: 0.54 and $\Delta E$ ratios of 1.0:0.58. Apparently the dependence of $\Delta F$ and $\Delta E$ upon $l$ becomes more linear as $M$ increases.

Results for the parallel-spring and square-network models suggest that the force drops and energy losses observed in a paper fiber network have an approximately linear dependence on a characteristic mesh length. The actual dependence, however, must be determined experimentally.

The effect of mesh length can also be deduced by a different argument as follows: Select a model $(M, N)$ for which calculations have been made so that $\bar{T}$ and $\bar{E}$ are known for a given $x$; i.e., $\bar{T}=\bar{T}(\delta), \bar{E}=\bar{E}(\delta)$. Select a mesh length $l$. The initial length of the model then is $2(N-1) l$. For each mesh length selected the model is elongated an amount $\Delta \mathrm{L}=2(N-1) l \delta$. This assures that the local tension at the bond break is always the same $(\mathrm{k} \delta)$. From eqs (1.1), (1.8) and (1.13)

$$
\begin{aligned}
& \Delta F=(2 M-1)(1-\bar{T}) k \delta \\
& \Delta E=2(N-1)(2 M-1) l\left(1-\frac{2 N-1}{2 N-2} \bar{E}\right) \cdot \frac{1}{2} k \delta^{2}
\end{aligned}
$$

TABLE 4. Force Drops $\Delta F$ and Energy Losses $\Delta E$ for Some Parallel-Spring Models. ${ }^{a}$

\begin{tabular}{c|c|c|c|c|c|c}
\hline \hline$M$ & $N$ & $l$ & $F_{b} / k \delta$ & $E_{b} / 0.5 k \delta^{2}$ & $\Delta F / k \delta$ & $\Delta E / 0.5 k \delta^{2}$ \\
\hline 11 & 11 & 1.0 & 21. & 420. & 0.1045 & .0424 \\
11 & 21 & 0.5 & 21. & 420. & .090 \\
21 & 21 & 0.5 & 41. & 820. & .0512 & 1.047 \\
11 & 41 & .25 & 21. & 420. & .0262 & 1.024 \\
21 & 41 & .25 & 41. & 820. & .0256 & 0.524 \\
\hline
\end{tabular}

${ }^{a}$ Initial length $L=2(N-1) l=20$. for all models.

TABle 5. Force Drops $\Delta F$ and Energy Losses $\Delta E$ for Some Square-Network Models. ${ }^{a}$

\begin{tabular}{c|c|c|c|c|c|c}
\hline \hline$M$ & $N$ & $l$ & $F_{b} / k \delta$ & $E_{b} / 0.5 k \delta^{2}$ & $\Delta F / k \delta$ & $\Delta E / 0.5 k \delta^{2}$ \\
\hline 11 & 11 & 1.0 & 21. & 420. & 0.5137 & 12.931 \\
11 & 21 & 0.5 & 21. & 420. & .2946 & 8.270 \\
21 & 21 & 0.5 & 41. & 820. & .2825 & 7.749 \\
11 & 41 & 0.25 & 21. & 420. & .1600 & 4.811 \\
21 & 41 & 0.25 & 21. & 820. & .1533 & 4.520 \\
\hline
\end{tabular}

${ }^{a}$ Initial length $L=2(N-1) l=20$. for all models. 
As $\bar{T}$ and $\bar{E}$ are functions of $\delta$ which is kept constant, $\Delta F$ is a constant independent of mesh length and $\Delta E$ is directly proportional to the mesh length. This conclusion is valid for either the parallel-spring or squarenetwork type of model.

The conclusion just obtained seems to conflict with that found by the analysis presented above. However in that analysis a number of models of different mesh length but having the same initial length were elongated, whereas in the present analysis the initial length of the model depends on the mesh length.

This latter deduction may be useful in experimental work, as illustrated in the following situation: Suppose that two handsheets have been manufactured from the same pulp stock. Let the first handsheet have twice the areal density as the second, so that its characteristic mesh length, is one-half as large. According to the analysis above, bond breaks in both handsheets should occur at the same force level, but a specimen from the second handsheet must be extended twice as much as one from the first in order to attain this force. Thus the average energy loss per bond break in the second handsheet should be twice that in the first. This suggests that energy parameters found by tests on handsheets of different areal density can be scaled to find the value corresponding to a standard mesh length.

\section{The Effect of Sample Size and Shape}

If several models, each with the same mesh length $l$, are elongated to the same tension, the force drops and energy losses are given by eqs (4.3) and (4.4). For square-network models it is necessary to know the appropriate values of $\bar{T}$ and $\bar{E}$ before the effects of sample size can be predicted. For parallel-spring models however, simple expressions for $\bar{T}$ and $\bar{E}$ are known, so that for large values of $M$ eqs (4.1) and (4.2) apply. Equation (4.1) predicts that the product of the force drop by the length of the specimen, $L \Delta F$, is a constant for all models having the same mesh length $l$, and eq (4.2) then states that the energy losses are constant.

Table 6 gives values of the force drop and energy loss for some parallel-spring models. Note that the quantities $L \Delta F / k \delta l$ and $\Delta E / 0.5 k \delta^{2} l$ have the same value in this table because of the choice of units. It is apparent that $\Delta F$ and $\Delta E$ for parallel-spring models are closely predicted by eqs (4.1) and (4.2). The three models for which $M=11$ (and width $W=2(M-1) l=20 l$ ) have values for $L \Delta F$ and $\Delta E$ of approximately 2.095 , and the two models for which $M=21(W=40 l$ ) have values of approximately 2.048 . Evidently $L \Delta F$ and $\Delta E$ approach a value of 2.0 with increasing $M$, as they should according to eqs (4.1) and (4.2).

Table 7 gives values of the force drop and energy loss for some square-network models. The values of $L \Delta F$ and $\Delta E$ for these models are roughly constant, but there seems to be some dependence upon both $M$ and $N$. For instance the three models for which $M=11(W=20 l)$ have lengths that increase in the ratio 20:40:80. The $L \Delta F$ values for these models are 10.27:11.79:12.80. The two models for which $M=21(W=40 l)$ have length ratios of 40:80 and $L \Delta F$ values of 11.30:12.27. Similar results are obtained for the $\Delta E$ values.

TABLE 6. Values of Force Drop $\Delta F$ and Energy Loss $\Delta E$ for Some Parallel-Spring Models.

\begin{tabular}{c|c|c|c|c|c}
\hline \hline$M$ & $N$ & $L / l$ & $\Delta F / k \delta$ & $L \Delta F / k \delta l$ & $\Delta E / 0.5 k \delta^{2} l$ \\
\hline 11 & 11 & 20 & 0.1045 & 2.090 & 2.090 \\
11 & 21 & 40 & .0524 & 2.095 & 2.095 \\
21 & 21 & 40 & .0512 & 2.047 & 2.047 \\
11 & 41 & 80 & .0262 & 2.097 & 2.097 \\
21 & 41 & 80 & .0256 & 2.049 & 2.049 \\
\hline
\end{tabular}

TABLE 7. Values of Force Drop $\Delta F$ and Energy Loss $\Delta E$ for Some Square-Network Models.

\begin{tabular}{l|l|c|c|c|c}
\hline \hline$M$ & $N$ & $L / l$ & $\Delta F / k \delta$ & $L \Delta F / k \delta l$ & $\Delta E / 0.5 k \delta^{2} l$ \\
\hline 11 & 11 & 20 & 0.5137 & 10.27 & 12.93 \\
11 & 21 & 40 & .2946 & 11.79 & 16.54 \\
21 & 21 & 40 & .2825 & 11.30 & 15.50 \\
11 & 41 & 80 & .1600 & 12.80 & 19.25 \\
21 & 41 & 80 & .1533 & 12.27 & 18.08 \\
\hline
\end{tabular}


TABLE 8. Values of $\Delta E / \Delta F$ for Some Square-Network Models.

\begin{tabular}{c|c|c|c|c}
\hline \hline$M$ & $N$ & $L / l$ & $\frac{1}{l \delta} \frac{\Delta E}{\Delta F}$ & $\frac{2}{L \delta} \frac{\Delta E}{\Delta F}$ \\
\hline 11 & 11 & 20 & 12.59 & 1.259 \\
11 & 21 & 40 & 28.07 & 1.403 \\
21 & 21 & 40 & 27.43 & 1.372 \\
11 & 41 & 80 & 60.14 & 1.503 \\
21 & 41 & 80 & 58.96 & 1.474 \\
\hline
\end{tabular}

The results for the square-network models suggest that various values of $L \Delta F$ and $\Delta E$ obtained by tests on specimens of the same characteristic mesh length but of different size and shape, are roughly comparable. For most accurate results however all test specimens should have the same standardized dimensions.

For the parallel-spring models, from eqs (1.1), (1.3), (1.8) and (1.12)

$$
\frac{\Delta E}{\Delta F}=\frac{E_{b}}{F_{b}}=\frac{2(N-1) l \delta}{2}=\frac{L \delta}{2}
$$

This equation states that for the parallel-spring models the ratio $\Delta E / \Delta F$ is proportional to the average elongation at which bond breaks occur, regardless of the value of $M$. Experimentally it is desirable to have significantly large force drops for a given energy loss, so the ratio $\Delta E / \Delta F$ should be kept small by making $L$ as small as feasible.

For the square-network models, from eqs (4.3) and (4.4)

$$
\frac{\Delta E}{\Delta F}=\left[\left(1-\frac{2 N-1}{2 N-2} \bar{E}\right) /(1-\bar{T})\right] \frac{L \delta}{2}
$$

Table 8 gives values for the ratio $\Delta E / \Delta F$ for some square-network models. Parallel-spring models have values of $\Delta E / \Delta F$ that are proportional to the initial length $L$ in accordance with eq (4.5), but for the squarenetwork models the dependence of $\Delta E / \Delta F$ upon $L$ is not quite linear, and there also seems to be a small dependence upon $M$. This is best shown by comparing values of the quantity $\frac{2}{L \delta}\left(\frac{\Delta E}{\Delta F}\right)$, which for parallelspring models is equal to unity but for the square-network models in table 8 varies between 1.2 and 1.5 depending upon the model.

Of most importance, however, is the confirmation that the quantity $\Delta E / \Delta F$ can be kept low by choosing a small value of $L$. This situation is limited somewhat in experimental tests. A specimen length at least twice the specimen width is preferred, in order to avoid excessive stress distortion near the clamps.

Although the results of tables 7 and 8 indicate that the values of $L \Delta F, \Delta E$ and $\Delta E / L \Delta F$ are insensitive to the value of $M$, there are circumstances where it is advantageous to vary the width of the specimen, as in the following experimental situation: Suppose that tests are being conducted on a given specimen, but the force drops observed are small, so that they cannot be measured with much certainty. The calculation of energy loss requires that the force drop be known as accurately as possible. Thus the experimental length should be small in order that the force drop be large. Further improvement is then achieved by decreasing the specimen width. This decreases the value of force at break $F_{b}$ without significantly affecting the value of the force drop. The amplification of the recording instrument can then be increased and the force drop better resolved.

\section{Dependence of Energy Loss Upon the Local Breaking Force.}

According to eq (4.4) the energy loss $\Delta E$ is proportional to the product of the quantity $\left(1-\frac{2 N-1}{2 N-2} \bar{E}\right)$ by the square of the local breaking force $k \delta$. For parallel-spring models $\bar{E}$ and therefore the quantity $\left(1-\frac{2 N-1}{2 N-2} \bar{E}\right)$ is independent of the value of this force. Thus for these models energy loss is proportional 


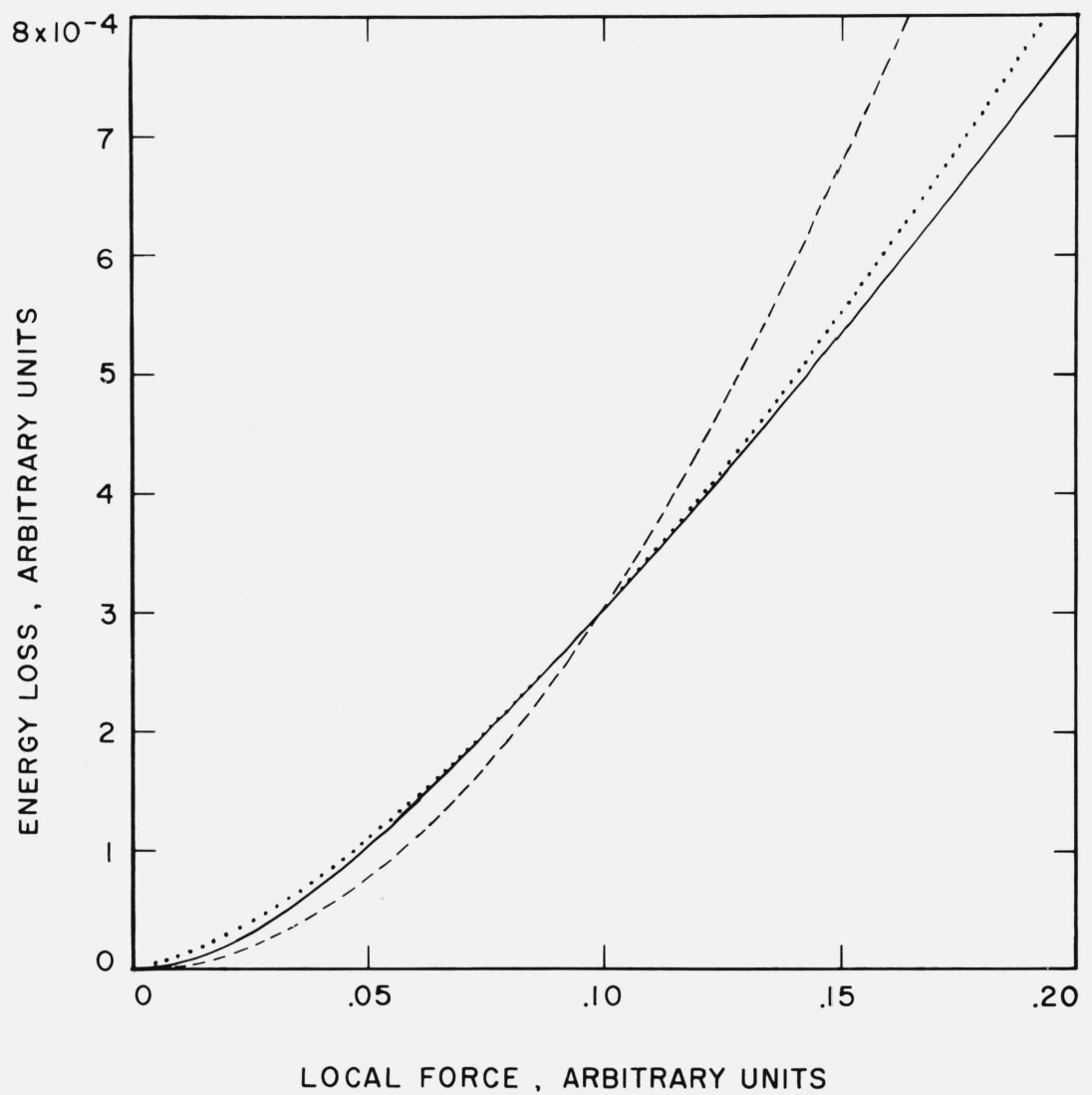

Figure 9. Curve of energy loss due to bond break vs breaking force for a square-network model for which $M=11, N=21$.

$\left(1-\frac{2 N-1}{2 N-2} \bar{E}\right) \delta^{2}$ is plotted vs elongation per unit length $\delta$, which is proportional to bond breaking force. Dashed line is plot of $K \delta^{2}$ vs $\delta$, and dotted line a plot of $K^{\prime} \delta^{1.5} \mathrm{vs} \delta$. The constants $K$ and $K^{\prime}$ are adjusted so that ordinates of the plots are equal at $\delta=0.10$.

to the square of the local force at break. For square-network models however the value of $\bar{E}$ depends upon the value of $\delta$, so this simple quadratic relationship no longer applies.

Figure 9 is a plot of the quantity $\left(1-\frac{2 N-1}{2 N-2} \bar{E}\right) \delta^{2}$ as a function of $\delta$. The ordinate in this case is proportional to the energy loss in a square-network model for which $M=11$ and $N=21$. This relationship, calculated from the data of table 3 , is shown by the solid line. A relationship having the same value for $\delta=0.10$, but for which the ordinate is of the form $K \delta^{2}$, where $K$ is a constant, is shown by the dashed line. A corresponding relationship of the form $K^{\prime} \delta^{1.5}$ is given by the dotted line. This latter relationship provides a better fit to the data than does the quadratic relationship.

The relationship between energy loss and local breaking force in an experimental test specimen is not known, and it does not seem feasible to determine it experimentally. It is possible that further studies with other more realistic mathematical models may provide more information on this relationship.

\section{Interpretation of the Energy Parameter.}

In the introduction an energy parameter $E$ for characterizing the adhesion between pulp fibers was described. This energy parameter is obtained experimentally by elongating thin web-like specimens in a sensitive tensile tester, and measuring the energy loss incurred each time a bond breaks, as denoted by a sharp drop in the force-elongation curve. The energy losses for a selected number of bond breaks are then averaged. 
The parameter $E$ is supposed to provide a measure of the adhesive force between fibers, but the previous discussion shows that $E$ depends on the structure of the specimen network as well. The meshes in a specimen network range over a variety of sizes, and the energy loss incurred by a break depends upon the size of the hole that opens up. If all the bonds in the neighborhood were of the same strength the parameter $E$ would roughly measure that strength, but could still take on a range of values depending on the size distribution of the meshes in the specimen network.

The force of adhesion between fibers in the network varies from bond to bond. It has just been shown that the energy loss incurred as the result of a bond break is not linearly related to the local breaking force at the bond. Therefore the average of a number of energy losses does not reflect a simple average of the local breaking forces.

Results of calculations with square-network models suggest that the energy loss has only an approximate linear relationship to the mesh length. This introduces a slight additional complication in the interpretation of what the $E$ parameter actually measures.

The interpretation of the $\mathrm{E}$ parameter may perhaps be better expressed by the following formulation: Let the energy losses be measured for each of a series of $n$ breaks, and let the energy loss $\Delta E_{i}$ for the $i^{\text {th }}$ break be given by

$$
\Delta E_{i}=K_{i} f_{i}^{\alpha} g\left(x_{i}\right)
$$

$K_{i}$ is a constant of the network that may vary from break to break if the network is significantly altered by the breaks. $f_{i}$ is the local force on the bond at break, and $\alpha$ is a constant having a value probably between 1 and 2. For a square-network model $\alpha$ has a value close to 1.5. $g\left(x_{i}\right)$ represents the functional dependence of $\Delta E_{i}$ upon a characteristic mesh length $x_{i}$, associated with the bond $i$. This relationship is probably almost a linear one.

Under these assumptions the characteristic energy $E$ is given by

$$
E=\frac{1}{n} \sum_{i=1}^{i=n} K_{i} f_{i}^{\alpha} g\left(x_{i}\right)
$$

This formula gives an approximate idea of the nature of the parameter $E$. However one should remember that this formula is based on the results of square-network model calculations, and that the square-network model is only a crude representation of the paper networks that are tested experimentally. It is likely that work with more appropriate models will provide an improved understanding of the nature of $E$.

\section{An Alternative Force Drop Parameter.}

Adhesion between pulp fibers could be characterized alternatively by means of a force drop parameter $F$. This parameter could be obtained by averaging force drops incurred in a series of bond breaks when a test specimen is elongated. The parameter $F$ has not been tested experimentally, but its use as a parameter to characterize adhesion seems feasible.

In order to calculate an energy parameter $E$ it is necessary to know the slope of the force-elongation curve in the vicinity of a force drop. The force drop parameter $F$ could be calculated more simply, as only the force drops are used.

In order that the force drop parameters be comparable, the test specimens must all have the same initial length. In other respects the force drop parameter $F$ is similar to the energy parameter $E$. Both parameters are sensitive to mesh size and mesh size distribution in the test specimen network. Neither parameter is linearly related to the average of the local bond breaking force. However the functional dependence of $F$ upon the average bond breaking force is different from that of $E$.

According to eq (4.3) the local force drop resulting from a bond break is proportional to the quantity $(1-\bar{T}) \delta$. A plot of $(1-\bar{T}) \delta$ vs $\delta$, which is proportional to the bond breaking force, is depicted in figure 8 for a square-network model with $M=11, N=21$. This curve can be fitted approximately by the curve $(1-\bar{T}) \delta \sim 0.0068716 \delta^{0.5}$. It can be inferred from this result that the parameter $F$ might not increase as rapidly with increase of local adhesion force as would be desired. Parameter $E$ with its possible $f^{1.5}$ dependence may be superior in this respect. 
The dependence of parameter $F$ upon local adhesive forces and upon mesh size is somewhat like that of parameter $E$. A suggested formula to express this dependence would be similar to eq (4.7) with different values for $K$ and $\alpha$, and a slightly different quasilinear function $g$.

\section{References}

[1] Smith, J. C. and Graminski, E. L. Characterizing the Interfiber Bond Strengths of Paper Pulps in Terms of a Breaking Energy, Progress report covering the period Jan. 1, 1976 to June 30, 1976, NBSIR 76-1148. Available from National Technical Information Service, PB 264,689.

[2] Smith, J. C. and Graminski, E. L. Characterizing the Interfiber Bond Strength of Paper Pulps in Terms of a Breaking Energy: Effect of Beating, Progress report covering the period July 1, 1976 through Sept. 30, 1976, NBSIR 77-1286. Available from National Technical Information Service, PB 276,473.

[3] Smith, J. C. and Graminski, E. L. Characterizing the Interfiber Bonding of Paper Pulps: Effect of Preparation Pressure on Tensile Test Specimens, Progress report covering the period Oct. 1, 1976 through March 31, 1977, NBSIR 78-1459. Available from National Technical Information Service, PB 280,279. 